\title{
An increase in the neutrophil-to-lymphocyte ratio during adjuvant chemotherapy indicates a poor prognosis in patients with stage II or III gastric cancer
}

Mikito Mori ${ }^{{ }^{*}}$, Kiyohiko Shuto ${ }^{1}$, Chihiro Kosugi ${ }^{1}$, Kazuo Narushima ${ }^{1}$, Hideki Hayashi ${ }^{2}$, Hisahiro Matsubara ${ }^{3}$ and Keiji Koda ${ }^{1}$

\begin{abstract}
Background: The neutrophil-to-lymphocyte ratio (NLR) and the platelet-to-lymphocyte ratio (PLR) are associated with poor prognoses in patients with gastric cancer; however, few studies have focused on the dynamic changes in these ratios during the treatment of patients with gastric cancer. Here, we assessed the clinical utility of changes in these ratios as prognostic indicators in patients with stage II or III gastric cancer who received adjuvant chemotherapy.

Methods: We retrospectively reviewed 100 patients who received S- 1 adjuvant chemotherapy at $\geq 70 \%$ of the relative dose intensity, and their NLRs and PLRs were evaluated at different times: prior to gastrectomy and upon commencement and termination of adjuvant chemotherapy. To assure the clinical utility of the changes in NLR and PLR as prognostic indicators, other clinical factors were assessed as well.

Results: Disease recurred in 35 patients as follows: lymph node metastasis (17 patients, 17.0\%), peritoneal metastasis (12 patients, 12.0\%), and hematogenous metastasis (6 patients, 6.0\%); 24 patients died. An increase in the NLR during adjuvant chemotherapy with S-1 was identified as an independent indicator associated with overall survival (hazard ratio [HR] 6.736, 95\% confidence interval $[\mathrm{Cl}] 2.420-18.748 ; P<0.001$ ), and relapse-free survival (HR 5.309, 95\%

Cl 2.585-10.901; $P<0.001)$.
\end{abstract}

Conclusion: An increase in the NLR during S-1 adjuvant chemotherapy may be a useful prognostic indicator in patients with stage II or III gastric cancer.

Keywords: Gastric cancer, Neutrophil-to-lymphocyte ratio, Platelet-to-lymphocyte ratio, Adjuvant chemotherapy, Overall survival, Relapse-free survival

\section{Background}

Gastric cancer (GC) is the fifth most common malignancy and the third leading cause of cancer-related death worldwide. Although the incidence of GC has declined in East Asia, GC is still the second most common cancer in Eastern Asia, including Japan [1]. In Japan, curative gastrectomy with D2 lymph-node dissection in patients with stage II or III GC is the main treatment for GC, and adjuvant therapy is also required

\footnotetext{
* Correspondence: m-mori@med.teikyo-u.ac.jp

${ }^{1}$ Department of Surgery, Teikyo University Chiba Medical Center, 3426-3

Anesaki, Ichihara, Chiba 299-0111, Japan

Full list of author information is available at the end of the article
}

to improve overall survival (OS) and relapse-free survival (RFS) [2].

Despite the effectiveness of adjuvant chemotherapy with S-1, further improvements in the treatments for patients with stage III GC are required, and approaches such as the development of combination chemotherapy $[3,4]$ and the analyses of molecular mechanisms [5] have been conducted. Several studies have focused on the relationship between systemic inflammation and cancer progression [6], and diverse prognostic scores based on systemic inflammatory variables have been assessed to predict prognosis in patients with cancer. In particular, the neutrophil-to-lymphocyte ratio (NLR) and

(c) The Author(s). 2018 Open Access This article is distributed under the terms of the Creative Commons Attribution 4.0 International License (http://creativecommons.org/licenses/by/4.0/), which permits unrestricted use, distribution, and reproduction in any medium, provided you give appropriate credit to the original author(s) and the source, provide a link to the Creative Commons license, and indicate if changes were made. The Creative Commons Public Domain Dedication waiver (http://creativecommons.org/publicdomain/zero/1.0/) applies to the data made available in this article, unless otherwise stated. 
platelet-to-lymphocyte ratio (PLR) are useful for predicting prognosis in patients with certain malignancies [79]. NLR and PLR are considered useful predictors of survival in patients with GC [10-12]. However, most of studies emphasize the importance of the pretreatment NLR and PLR for GC, and few reports discuss the importance of the change in the NLR and PLR after treatment [13-15]. To assess how the changes in NLR and PLR reflected prognoses of patents with stage II or III GC who received adjuvant chemotherapy, we investigated the relationship between clinical factors such as change in the NLR or PLR during adjuvant chemotherapy and the survival of patients with stage II or III GC.

\section{Methods}

\section{Patients}

One hundred and eighteen patients were histologically diagnosed with stage II or III GC after curative gastrectomy with D2 lymph-node dissection between January 2006 and January 2017 at Teikyo University Chiba Medical Center and Chiba University Hospital. Pathological staging was performed according to the cancer staging system for GC recommended in the 8th edition of the American Joint Committee on Cancer (AJCC) Cancer Staging Manual. In the analysis, there were no patients who died within 30 days after surgery, died of causes unrelated to cancer, had other malignancies, or had inflammatory diseases. To eliminate potential effects on relapse and survival, 18 out of 118 patients were excluded for the following reasons: 10 patients treated with neoadjuvant chemotherapy and 8 patients treated with S-1 adjuvant chemotherapy at less than $70 \%$ of the relative dose intensity (RDI). Therefore, we retrospectively reviewed 100 patients who received S-1 adjuvant chemotherapy at greater than $70 \%$ of the RDI for 1 year or until tumor recurrence.

\section{Treatment}

After curative gastrectomy, all patients received adjuvant chemotherapy with S-1 (TS-1, Taiho Pharmaceutical, Tokyo, Japan), which is an orally active preparation combining tegafur, gimeracil, and oteracil in a molar ratio of 1:0.4:1. S-1 (80-120 mg per day) was administered for 4 weeks followed by a 2 -week rest or for 2 weeks followed by a 1 -week rest. The daily dose of $S-1$ was determined based on body surface area. This 3- or 6-week cycle was repeated for 1 year or until a tumor recurrence was objectively diagnosed.

\section{Evaluation of the NLR and PLR}

A routine blood examination was performed before curative gastrectomy and during adjuvant chemotherapy. The NLR or PLR was calculated by dividing the lymphocyte count into neutrophil or platelet count. The pNLR, iNLR, and fNLR (NLRs), were defined as follows: the preoperative NLR (pNLR), the NLR on the initial day of adjuvant chemotherapy divided by the preoperative NLR (iNLR), and the NLR on the final day of adjuvant chemotherapy divided by the NLR on the initial day of adjuvant chemotherapy (fNLR), respectively. The pPLR, iPLR, and fPLR (PLRs) were similarly defined. Patients were divided into two groups according to a cutoff value. For the pNLR and pPLR, the median was defined as the cutoff value. The patient was classified as positive pNLR or positive pPLR when the PNLR or pPLR was greater than the median (pNLR or pPLR $\geq$ the median). The patient was classified as negative pNLR or negative pPLR when the pNLR or pPLR was less than the median (pNLR or pPLR < the median). One was defined as the cutoff value for the other NLRs and PLRs such as iNLR, iPLR, fNLR, and fPLR. Furthermore, the patient was classified as positive NLR or positive PLR when the NLR or PLR was $\geq 1$ (NLR or PLR $\geq 1$ ). The patient was classified as negative NLR or negative PLR when the NLR or PLR was $<1($ NLR or PLR $<1)$.

\section{Statistical analysis}

The relationships between clinical factors and NLRs or PLRs were analyzed using Fisher's exact test. OS and RFS curves were generated using the Kaplan-Meier method, and univariate analysis of survival was performed using the log-rank test. Multivariate analysis was performed using a Cox proportional-hazards model to determine the statistical significance of prognostic factors. $P$ values in multiple comparisons were corrected using a false discovery rate. All statistical analyses were performed using SPSS for Windows (version 20.0, IBM Corp., Armonk, NY, USA).

\section{Results}

The clinical characteristics of 100 patients (27 women and 73 men) with stage II or III GC who received adjuvant chemotherapy with S-1 are summarized in Table 1 . The median age was 66 years (range, 36-82 years), including 41 patients $<65$ years and 59 patients $\geq 65$ years. The median tumor size was $60 \mathrm{~mm}$ (range, 15-170 mm), including 48 patients with tumors $<60 \mathrm{~mm}$ and 52 patients with tumors $\geq 60 \mathrm{~mm}$. The tumor cells of 35 and 65 patients were histologically classified as differentiated and undifferentiated, respectively. Pathological tumor (pT) stages were as follows: 5 patients, pT1; 12 patients, pT2; 41 patients, pT3; and 42 patients, pT4. Pathological nodal (pN) stages were as follows: 14 patients, pN0; 25 patients, pN1; 31 patients, pN2; and 30 patients, pN3. Thirty-nine patients were diagnosed with pathological cancer stage (pStage) II GC and 61 patients were diagnosed with pStage III GC.

The median pNLR was 2.6 (range, 0.8-9.8), and the median pPLR was 149.4 (range, 67.7-555.3). Fifty patients were classified as positive pNLR or PPLR, and 50 patients were classified as negative pNLR or pPLR. 
Table 1 Demographics of GC patients treated with S-1 adjuvant chemotherapy

\begin{tabular}{|c|c|}
\hline Factors & $N=100$ \\
\hline $\operatorname{Sex}(M / F)$ & $73 / 27$ \\
\hline Age (<65/ $\geq 65$ years) & $41 / 59$ \\
\hline Tumor size $(<60 / \geq 60 \mathrm{~mm})$ & $48 / 52$ \\
\hline Histologic type (Diff/Undiff) & $35 / 65$ \\
\hline $\mathrm{pT}(1 / 2 / 3 / 4)$ & $5 / 12 / 41 / 42$ \\
\hline $\mathrm{pN}(0 / 1 / 2 / 3)$ & $14 / 25 / 31 / 30$ \\
\hline pStage (II/III) & $39 / 61$ \\
\hline Lymphatic invasion (+/-) & $80 / 20$ \\
\hline Venous invasion (+/-) & $78 / 22$ \\
\hline pNLR (+/-) & $50 / 50$ \\
\hline iNLR (+/-) & $26 / 74$ \\
\hline fNLR $(+/-)$ & $38 / 62$ \\
\hline pPLR (+/-) & $50 / 50$ \\
\hline $\operatorname{iPLR}(+/-)$ & $50 / 50$ \\
\hline fPLR (+/-) & $35 / 65$ \\
\hline Recurrence (+/-) & $35 / 65$ \\
\hline Site of relapse (H/P/LYM/Lo) & $6 / 12 / 17 / 2$ \\
\hline Outcome (D/A) & $24 / 76$ \\
\hline
\end{tabular}

$M$, male; F, female; Diff, differentiated type; Undiff, undifferentiated type; $\mathrm{pT}$, $\mathrm{pN}$, pStage = pathological $\mathrm{T}$ stage, N stage. Pathological cancer stage according to the 8th edition of the American Joint Committee on Cancer (AJCC) Cancer Staging Manual; pNLR or pPLR, preoperative neutrophil or platelet-to-lymphocyte ratio; iNLR and $\mathrm{PLR}$, the ratio of the NLR or PLR on the initial day of adjuvant chemotherapy to the $\mathrm{PNLR}$ or $\mathrm{PPLR}$; fNLR and FPLR, the ratio of the NLR or PLR on the final day of adjuvant chemotherapy to the iNLR or iPLR; $H$, hematogenous metastasis; $P$, peritoneal metastasis; $L Y M$, lymph node metastasis; Lo, local recurrence; $D / A$, dead or alive

Thirty-eight and 62 patients were classified as positive fNLR and negative fNLR, respectively. Thirty-five and 65 patients were classified as positive and negative fPLR, respectively. Thirty-five patients developed recurrences as follows: lymph node metastasis (17 patients, $17.0 \%$ ), peritoneal metastasis (12 patients, $12.0 \%$ ), hematogenous metastasis (6 patients, $6.0 \%$ ), and local recurrence ( 2 patients, $2 \%)$. Twenty-four patients died of GC during the median follow-up period of 37.1 months (range, 5.3-108.8 months).

To evaluate whether the NLR or PLR may serve as a useful indicator of OS and RFS, all NLRs and PLRs were assessed using receiver operating characteristic (ROC) curves. In the analysis of OS, the area under the curves (AUCs) of pNLR, iNLR, and fNLR were 0.659 (sensitivity, 70.8\%; specificity, 56.6\%), 0.463 (sensitivity, 33.3\%; specificity, 76.3\%), and 0.748 (sensitivity, $75.0 \%$; specificity, 69.7\%), respectively. The AUCs of pPLR, iPLR, and fPLR were 0.666 (sensitivity, 66.7\%; specificity, 55.3\%), 0.446 (sensitivity, 41.7\%; specificity, $47.4 \%$ ), and 0.708 (sensitivity, 58.3\%; specificity, 72.4\%), respectively. For RFS, the AUCs of pNLR, iNLR, and fNLR were 0.619 (sensitivity, 62.9\%; specificity, 56.9\%), 0.453 (sensitivity,
25.7\%; specificity, 73.8\%), and 0.706 (sensitivity, 68.6\%; specificity, $73.8 \%$ ), respectively. The AUCs of pPLR, iPLR, and fPLR were 0.605 (sensitivity, 54.3\%; specificity, 52.3\%), 0.447 (sensitivity, 40.0\%; specificity, 44.6\%), and 0.603 (sensitivity, $42.9 \%$; specificity, $69.2 \%$ ), respectively.

The ROC curves suggested that fNLR was the best prognostic indicator of NLRs and PLRs (Fig. 1). There was no significant difference between positive and negative pNLRs or iNLRs. In contrast, there was a significant difference in recurrences and outcomes between positive and negative fNLRs (Table 2). There was no significant difference between positive and negative iPLRs or fPLRs. However, there was a significant difference between positive and negative pPLRs associated with pT and pStage (Table 3). Univariate analysis of OS revealed that tumor size $(P=0.008)$, histological type $(P=0.005)$, fNLR $(P<0.001)$, and PPLR $(P=0.004)$ were associated with a shorter OS.

Multivariate analysis of the significant variables identified using univariate analysis of OS revealed that tumor size (HR 3.115, 95\% CI 1.230-7.889; $P=0.017$ ), histological type (HR 4.472, 95\% CI 1.308-15.287; $P=0.017$ ) and fNLR (HR 6.736, 95\% CI 2.420-18.748; $P<0.001$ ) were independently associated with a shorter OS, and the ANLR was identified as a significant indicator of OS (Table 4). In univariate analysis of RFS, fNLR $(P<0.001)$ was only associated with a shorter RFS. Multivariate analysis of the significant variables identified by univariate analysis of RFS revealed that fNLR (HR 5.309, 95\% CI $2.585-10.901 ; P<0.001)$ was independently associated with a shorter RFS, and fNLR was identified as a significant indicator of RFS (Table 5). We suggest, therefore, that the fNLR may be the best prognostic value for patients during adjuvant chemotherapy with S-1 (Fig. 2).

\section{Discussion}

There is a significant relationship between inflammation and cancer, as hypothesized by Rudolf Virchow [16]. The relationship between the NLR and survival is complicated, and the precise mechanisms are unknown. Generally, neutrophils are the most common leukocyte subset in human peripheral blood, accounting for $50-70 \%$ of total circulating leukocytes. Furthermore, neutrophils are considered essential for protecting the host and for the development of cancer-associated inflammation, because neutrophils are thought to release cytokines, chemokines, and granule proteins, which produce a favorable microenvironment for tumor growth and promote tumor progression $[6,17]$. In contrast, lymphocytes play a vital role in suppressing tumor development, and the diverse functions of lymphocytes may be related to protection against the development and progression of cancer $[18,19]$.

Based on the inverse relationship between neutrophils and lymphocytes, the NLR is considered to provide 

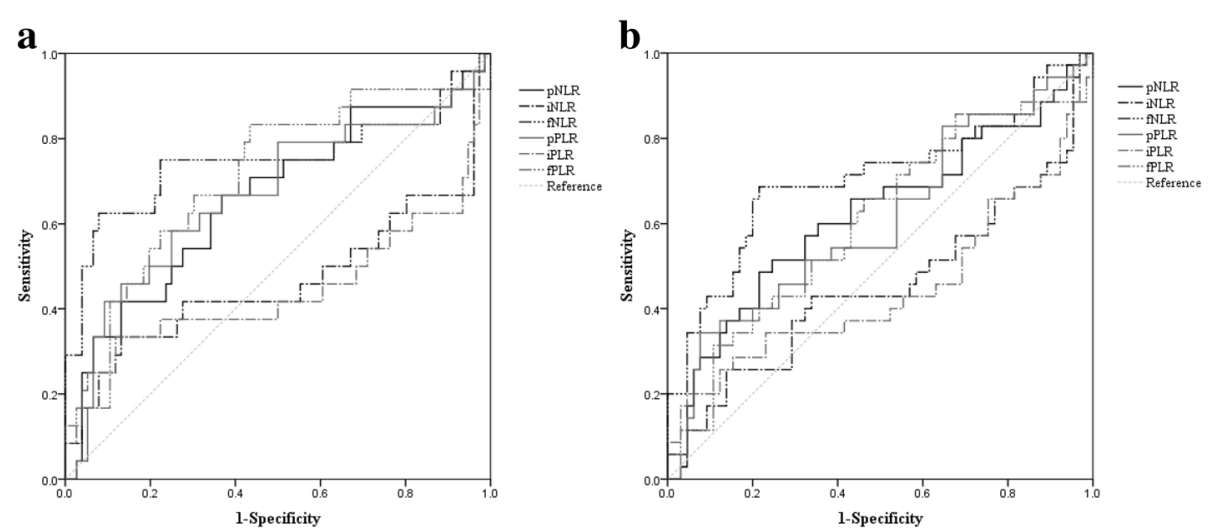

Fig. 1 Evaluation of NLRs and PLRs as prognostic indicators using receiver operating characteristic curves. a overall survival, $\mathbf{b}$ relapse-free survival

useful information related to cancer progression. A representative study assessing the relationship between pretreatment NLRs and survival in 1028 patients with primary GC who underwent gastrectomy was performed by Shimada et al. Their data suggested that a high preoperative NLR serves as an independent risk factor for OS [11]. Jung et al. evaluated 293 patients who had undergone curative gastrectomy. Their analysis established that a high preoperative NLR was significantly related to a poor OS or disease-free survival in patients with stage III or IV GC [12].

With advances in chemotherapy, several studies have focused on pretreatment NLRs and PLRs as useful predictors of response to chemotherapy in patients with certain malignancies [20-22]. In the analysis of pretreatment NLRs or PLRs, an important potential limitation was that cutoff values for NLRs or PLRs differed among those studies, although their results indicated a significant association between high blood-neutrophil counts and poor clinical outcomes in GC.
Studies focused on the dynamic changes in the NLR after treatment showed that the change in the NLR in patients with renal cell carcinoma was associated with outcomes and clinicopathological parameters [23, 24]. Moreover, the change in the NLR was a more statistically robust predictor of OS in patients with non-small cell lung cancer or urothelial carcinoma compared with the pretreatment NLR $[25,26]$. Although most studies in GC have focused on pretreatment NLRs or PLRs, few reports have focused on the dynamic change in the NLR or PLR after treatment in GC [13-15]. Indeed, few studies have focused on the change in the NLR during chemotherapy administered to patients with advanced GC. For example, Lee et al. found that the NLR, PLR, and changes in their values served as independent prognostic indicators of OS in patients with unresectable and recurrent GC who received FOLFOX chemotherapy [13]. Jin et al. suggested that the NLR was a potential predictor of survival in patients with stage III or IV GC

Table 2 Relationship between NLRs and clinical factors

\begin{tabular}{|c|c|c|c|c|c|c|c|c|c|}
\hline Factor & pNLR (+) & pNLR (-) & $P$-value & iNLR (+) & iNLR (-) & $P$-value & fNLR (+) & fNLR (-) & $P$-value \\
\hline $\operatorname{Sex}(M / F)$ & $34 / 16$ & $39 / 11$ & 0.368 & $23 / 3$ & $50 / 24$ & 0.043 & $28 / 10$ & $45 / 17$ & 1.000 \\
\hline Age (<65/ $\geq 65$ years $)$ & $21 / 29$ & $20 / 30$ & 1.000 & $11 / 15$ & $30 / 44$ & 0.875 & $16 / 22$ & $25 / 37$ & 1.000 \\
\hline Tumor size $(<60 / \geq 60 \mathrm{~mm})$ & $20 / 30$ & $28 / 22$ & 0.161 & $14 / 12$ & $34 / 40$ & 0.504 & $16 / 22$ & $32 / 30$ & 0.412 \\
\hline Histologic type (Diff/Undiff) & $15 / 35$ & $20 / 30$ & 0.402 & $8 / 18$ & $27 / 47$ & 0.644 & $11 / 27$ & $24 / 38$ & 0.390 \\
\hline pT (1/2/3/4) & $1 / 6 / 18 / 25$ & $4 / 6 / 23 / 17$ & 0.275 & $2 / 4 / 10 / 10$ & $3 / 8 / 31 / 32$ & 0.731 & $2 / 7 / 11 / 18$ & $3 / 5 / 30 / 24$ & 0.180 \\
\hline $\mathrm{pN}(0 / 1 / 2 / 3)$ & $4 / 11 / 14 / 21$ & $10 / 14 / 17 / 9$ & 0.045 & $2 / 11 / 9 / 4$ & $12 / 14 / 22 / 26$ & 0.056 & $3 / 9 / 14 / 12$ & $11 / 16 / 17 / 18$ & 0.510 \\
\hline pStage (II/III) & $13 / 37$ & $26 / 24$ & 0.013 & $12 / 14$ & $27 / 47$ & 0.484 & $15 / 23$ & $24 / 38$ & 1.000 \\
\hline Lymphatic invasion (+/-) & $43 / 7$ & $37 / 13$ & 0.211 & $21 / 5$ & $59 / 15$ & 1.000 & $28 / 10$ & $52 / 10$ & 0.303 \\
\hline Venous invasion (+/-) & $40 / 10$ & $38 / 12$ & 0.810 & $20 / 6$ & $58 / 16$ & 1.000 & $30 / 8$ & $48 / 14$ & 1.000 \\
\hline Recurrence (+/-) & $22 / 28$ & $13 / 37$ & 0.093 & 9/17 & $26 / 48$ & 1.000 & $24 / 14$ & $11 / 51$ & $<0.001^{*}$ \\
\hline Outcome (D/A) & $17 / 33$ & $7 / 43$ & 0.034 & $8 / 18$ & $16 / 58$ & 0.425 & $18 / 20$ & $6 / 56$ & $<0.001^{*}$ \\
\hline
\end{tabular}

$\mathrm{M}$, male; F, female; Diff, differentiated type; Undiff, undifferentiated type; $\mathrm{pT}$, pN, pStage = pathological T stage, $\mathrm{N}$ stage. Pathological cancer stage according to the 8th edition of the American Joint Committee on Cancer (AJCC) Cancer Staging Manual; pNLR, preoperative neutrophil-to-lymphocyte ratio; iNLR, the ratio of the NLR on the initial day of adjuvant chemotherapy to the pNLR; fNLR, the ratio of the NLR on the final day of adjuvant chemotherapy to the iNLR; D/A, dead or alive; ${ }^{*}$-value indicates statistical significance after false discovery rate correction 
Table 3 Relationship between PLRs and clinical factors

\begin{tabular}{|c|c|c|c|c|c|c|c|c|c|}
\hline Factor & pPLR $(+)$ & pPLR (-) & P-value & iPLR (+) & iPLR (-) & P-value & fPLR (+) & fPLR (-) & $P$-value \\
\hline$\overline{S e x(M / F)}$ & $32 / 18$ & $41 / 9$ & 0.070 & $39 / 11$ & $34 / 16$ & 0.368 & $24 / 11$ & $49 / 16$ & 0.486 \\
\hline Age (<65/ $\geq 65$ years) & $24 / 26$ & $17 / 33$ & 0.222 & $21 / 29$ & $20 / 30$ & 1.000 & $15 / 20$ & $26 / 39$ & 0.833 \\
\hline Tumor size $(<60 / \geq 60 \mathrm{~mm})$ & $21 / 29$ & $27 / 23$ & 0.317 & $26 / 24$ & $22 / 28$ & 0.548 & $15 / 20$ & $33 / 32$ & 0.531 \\
\hline Histologic type (Diff/Undiff) & $15 / 35$ & $20 / 30$ & 0.402 & $19 / 31$ & $16 / 34$ & 0.675 & $8 / 27$ & $27 / 38$ & 0.080 \\
\hline pT (1/2/3/4) & $0 / 6 / 16 / 28$ & $5 / 6 / 25 / 14$ & $0.006^{*}$ & $4 / 8 / 21 / 17$ & $1 / 4 / 20 / 25$ & 0.210 & $3 / 5 / 11 / 16$ & $2 / 7 / 30 / 26$ & 0.393 \\
\hline $\mathrm{pN}(0 / 1 / 2 / 3)$ & $4 / 13 / 12 / 21$ & $10 / 12 / 19 / 9$ & 0.030 & $8 / 15 / 16 / 11$ & 6/10/15/19 & 0.332 & $4 / 6 / 13 / 12$ & 10/19/18/18 & 0.480 \\
\hline pStage (II/III) & $12 / 38$ & $27 / 23$ & $0.004^{*}$ & $25 / 25$ & $14 / 36$ & 0.040 & $13 / 22$ & $26 / 39$ & 0.832 \\
\hline Lymphatic invasion (+/-) & $42 / 8$ & $38 / 12$ & 0.454 & $39 / 11$ & $41 / 9$ & 0.803 & $26 / 9$ & $54 / 11$ & 0.307 \\
\hline Venous invasion (+/-) & $39 / 11$ & $39 / 11$ & 1.000 & $38 / 12$ & $40 / 10$ & 0.810 & $25 / 10$ & $53 / 12$ & 0.312 \\
\hline Recurrence (+/-) & $19 / 31$ & $16 / 34$ & 0.675 & $14 / 36$ & $21 / 29$ & 0.208 & $15 / 20$ & $20 / 45$ & 0.274 \\
\hline Outcome (D/A) & $16 / 34$ & $8 / 42$ & 0.100 & $10 / 40$ & $14 / 36$ & 0.483 & $14 / 21$ & $10 / 55$ & 0.013 \\
\hline
\end{tabular}

$\mathrm{M}$, male; F, female; Diff, differentiated type; Undiff, undifferentiated type; $\mathrm{pT}$, pN, pStage = pathological T stage, N stage. Pathological cancer stage according to the 8th edition of the American Joint Committee on Cancer (AJCC) Cancer Staging Manual; pPLR, preoperative platelet-to-lymphocyte ratio; iPLR, the ratio of the PLR on the initial day of adjuvant chemotherapy to $\mathrm{PPLR}$; $\mathrm{PLR}$, the ratio of the PLR on the final day of adjuvant chemotherapy to iPLR; D/A, dead or alive; * $P$-value indicates statistical significance after false discovery rate correction

who received neoadjuvant chemotherapy [14]. Therefore, the change in the NLR associated with treatment was a more meaningful measurement than that provided by the pretreatment NLR, because the change in the NLR may reflect a dynamic reaction of the immune response caused by the treatment.

We selectively analyzed 100 patients who received S-1 adjuvant chemotherapy at greater than $70 \%$ of the RDI. In view of the RDI, some studies have demonstrated that insufficient RDI of chemotherapy was related to a poor prognosis in some malignancies such as breast, ovarian, colon, and pancreatic cancers [27-30]. In GC, two studies suggested that a decreased RDI of S-1 will lessen the efficacy of S-1 adjuvant chemotherapy for GC and may lead to a poor prognosis [31,32]. Our present study included only patients with stage II or III GC who received sufficient adjuvant chemotherapy with S-1 after surgery and excluded patients with very advanced metastatic

Table 4 Relationship between clinical factors and OS in GC patients treated with S-1 adjuvant chemotherapy

\begin{tabular}{|c|c|c|c|c|}
\hline \multirow[t]{2}{*}{ Factors } & \multirow[t]{2}{*}{$N=100$} & \multirow{2}{*}{$\begin{array}{l}\text { Univariate analysis } \\
\text { P-valuea }\end{array}$} & \multicolumn{2}{|l|}{ Multivariate analysis } \\
\hline & & & $\mathrm{HR}(95 \% \mathrm{Cl}) \mathrm{b}$ & P-valueb \\
\hline $\operatorname{Sex}(M / F)$ & $73 / 27$ & 0.417 & & \\
\hline Age $(<65 / \geq 65$ years $)$ & $41 / 59$ & 0.558 & & \\
\hline Tumor size $(<60 / \geq 60 \mathrm{~mm})$ & $48 / 52$ & $0.008^{*}$ & 3.115 (1.230-7.889) & 0.017 \\
\hline Histologic type (Diff/Undiff) & $35 / 65$ & $0.005^{*}$ & $4.472(1.308-15.287)$ & 0.017 \\
\hline pT $(1 / 2 / 3 / 4)$ & $5 / 12 / 41 / 42$ & 0.366 & & \\
\hline $\mathrm{pN}(0 / 1 / 2 / 3)$ & $14 / 25 / 31 / 30$ & 0.023 & & \\
\hline pStage (I/III) & $39 / 61$ & 0.043 & & \\
\hline pNLR (+/-) & $50 / 50$ & 0.018 & & \\
\hline pPLR (+/-) & $50 / 50$ & 0.074 & & \\
\hline iNLR (+/-) & $26 / 74$ & 0.455 & & \\
\hline iPLR (+/-) & $50 / 50$ & 0.308 & & \\
\hline fNLR $(+/-)$ & $38 / 62$ & $<0.001^{*}$ & $6.736(2.420-18.748)$ & $<0.001$ \\
\hline $\mathrm{fPLR}(+/-)$ & $35 / 65$ & $0.004^{*}$ & & \\
\hline CEA $(<5.0 / \geq 5.0 \mathrm{ng} / \mathrm{ml})$ & $26 / 74$ & 0.118 & & \\
\hline CA19-9 $(<37.0 / \geq 37.0 \mathrm{U} / \mathrm{ml})$ & $18 / 82$ & 0.262 & & \\
\hline
\end{tabular}

$\mathrm{M}$, male; F, female; Diff, differentiated type; Undiff, undifferentiated type; $\mathrm{pT}$, pN, pStage = pathological T stage, N stage. Pathological cancer stage according to the 8th edition of the American Joint Committee on Cancer (AJCC) Cancer Staging Manual; pNLR or pPLR, preoperative neutrophil or platelet-to-lymphocyte ratio; iNLR and iPLR, the ratio of the NLR or PLR on the initial day of adjuvant chemotherapy to the pNLR or pPLR; fNLR and fPLR, the ratio of the NLR or PLR on the final day of adjuvant chemotherapy to the iNLR or iPLR; CEA, carcinoembryonic antigen; CA19-9, carbohydrate antigen 19-9; ${ }^{a}$ Log-rank test; ${ }^{b}$ Cox proportional hazards model; * $P$-value indicates statistical significance after false discovery rate correction 
Table 5 Relationship between clinical factors and RFS in GC patients treated with S-1 adjuvant chemotherapy

\begin{tabular}{|c|c|c|c|c|}
\hline \multirow[t]{2}{*}{ Factors } & \multirow[t]{2}{*}{$N=100$} & \multirow{2}{*}{$\begin{array}{l}\text { Univariate analysis } \\
\text { P-valuea }\end{array}$} & \multicolumn{2}{|l|}{ Multivariate analysis } \\
\hline & & & $\mathrm{HR}(95 \% \mathrm{Cl}) \mathrm{b}$ & P-valueb \\
\hline $\operatorname{Sex}(M / F)$ & $73 / 27$ & 0.821 & & \\
\hline Age $(<65 / \geq 65$ years $)$ & $41 / 59$ & 0.558 & & \\
\hline Tumor size $(<60 / \geq 60 \mathrm{~mm})$ & $48 / 52$ & 0.093 & & \\
\hline Histologic type (Diff/Undiff) & $35 / 65$ & 0.199 & & \\
\hline pT $(1 / 2 / 3 / 4)$ & $5 / 12 / 41 / 42$ & 0.226 & & \\
\hline $\mathrm{pN}(0 / 1 / 2 / 3)$ & $14 / 25 / 31 / 30$ & 0.014 & & \\
\hline pStage (I/III) & $39 / 61$ & 0.016 & & \\
\hline pNLR (+/-) & $50 / 50$ & 0.057 & & \\
\hline $\operatorname{pPLR}(+/-)$ & $50 / 50$ & 0.494 & & \\
\hline iNLR (+/-) & $26 / 74$ & 0.965 & & \\
\hline $\operatorname{iPLR}(+/-)$ & $50 / 50$ & 0.204 & & \\
\hline fNLR $(+/-)$ & $38 / 62$ & $<0.001^{*}$ & $5.309(2.585-10.901)$ & $<0.001$ \\
\hline fPLR (+/-) & $35 / 65$ & 0.144 & & \\
\hline CEA $(<5.0 / \geq 5.0 \mathrm{ng} / \mathrm{ml})$ & $26 / 74$ & 0.262 & & \\
\hline CA19-9 $(<37.0 / \geq 37.0 \mathrm{U} / \mathrm{ml})$ & 18/82 & 0.055 & & \\
\hline
\end{tabular}

$\mathrm{M}$, male; F, female; Diff, differentiated type; Undiff undifferentiated type; $\mathrm{pT}$, pN, pStage = pathological T stage, N stage. Pathological cancer stage according to the 8th edition of the American Joint Committee on Cancer (AJCC) Cancer Staging Manual; pNLR or pPLR, preoperative neutrophil or platelet-to-lymphocyte ratio; iNLR and iPLR, the ratio of the NLR or PLR on the initial day of adjuvant chemotherapy to the pNLR or pPLR; fNLR and fPLR, the ratio of the NLR or PLR on the final day of adjuvant chemotherapy to the iNLR or iPLR; CEA, carcinoembryonic antigen; CA19-9, carbohydrate antigen $19-9$; ${ }^{a}$ Log-rank test; ${ }^{\text {b } C o x ~ p r o p o r t i o n a l ~ h a z a r d s ~ m o d e l ; ~}{ }^{*} P$-value indicates statistical significance after false discovery rate correction

disease. Furthermore, the effect on neoadjuvant chemoradiation therapy may bias the results. Although our current findings should be interpreted with caution because we performed a retrospective analysis of a small number of patients, and additional assessments are required to elucidate the relationship between the NLR and systemic inflammatory or immune responses at the time of recurrence, we believe that its potential clinical significance justifies further investigation.

\section{Conclusions}

In conclusion, our study demonstrated that fNLR was a better prognostic indicator compared with PNLR in patients with stage II or III GC who received sufficient adjuvant chemotherapy with $\mathrm{S}-1$. The change in the NLR during adjuvant chemotherapy with S-1 may be easier to determine, less expensive to measure, and useful for indicating a poor prognosis in patients with stage II or III GC.
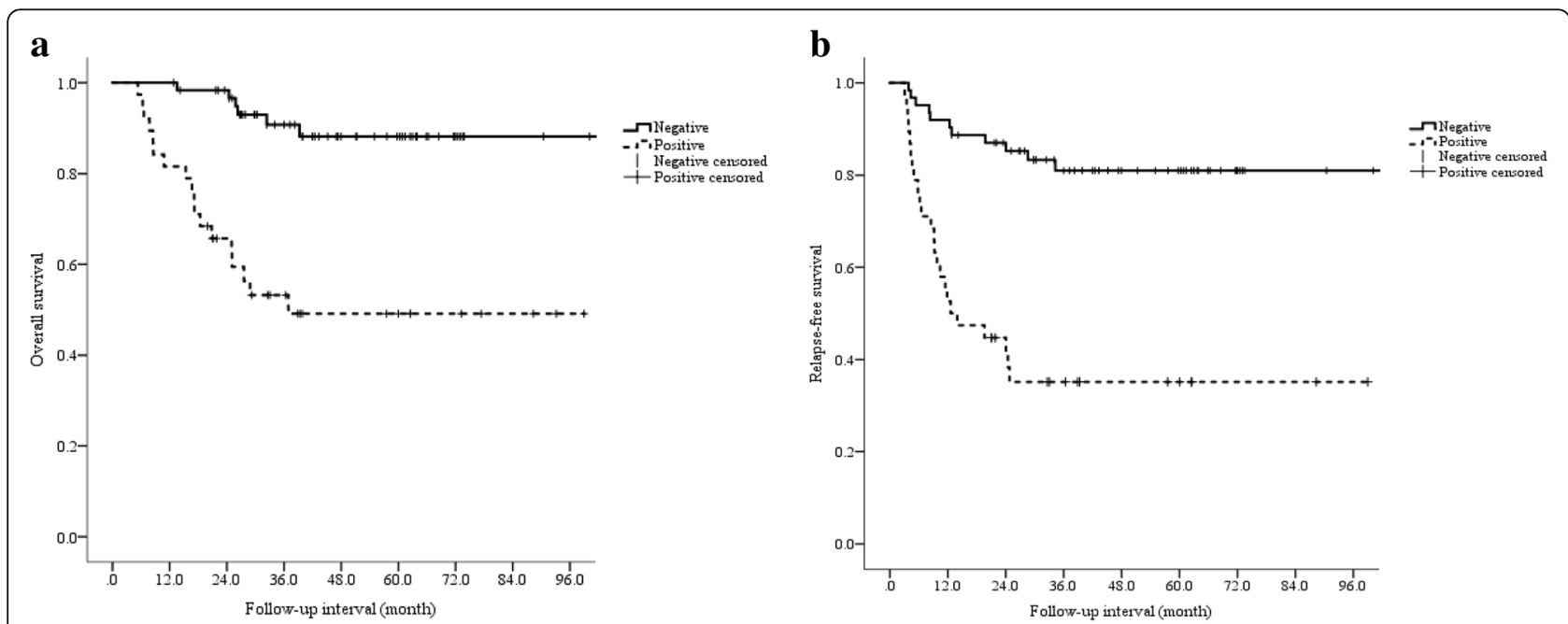

Fig. 2 Survival curves of positive and negative fNLR values using the log-rank test. a overall survival $(P<0.001)$, b relapse-free survival $(P<0.001)$ 


\section{Abbreviations}

AUC: Area under the ROC curve; fNLR: The ratio of the NLR on the final day of adjuvant chemotherapy to the NLR on the initial day of adjuvant chemotherapy; fPLR: The ratio of the PLR on the final day of adjuvant chemotherapy to the PLR on the initial day of adjuvant chemotherapy; GC: Gastric cancer; iNLR: The ratio of the NLR on the initial day of adjuvant chemotherapy to the preoperative NLR; iPLR: The ratio of the PLR on the initial day of adjuvant chemotherapy to the preoperative PLR; NLR: Neutrophilto-lymphocyte ratio; OS: Overall survival; PLR: Platelet-to-lymphocyte ratio; pNLR: Preoperative NLR; pPLR: Preoperative PLR; RFS: Relapse-free survival; ROC: Receiver operating characteristics

\section{Acknowledgments}

We thank Mark Abramovitz, Ph.D., from Edanz Group (http://

www.edanzediting.com/jp) for editing a draft of this manuscript.

\section{Funding}

Not applicable.

\section{Availability of data and materials}

The datasets used and/or analyzed during the current study are available from the corresponding author upon reasonable request.

\section{Authors' contributions}

SK, MM, KC, and NK collected the data. KC, MM, and HH performed the statistical analysis. $\mathrm{MH}, \mathrm{KK}$, and MM drafted the manuscript. All authors read and approved the final manuscript. All authors read and approved the final manuscript.

\section{Authors' information}

KK is a professor in the Department of Surgery, Teikyo University Chiba Medical Center, Chiba, Japan. HH is a professor in the Center for Frontier Medical Engineering, Chiba University, Chiba, Japan. $\mathrm{MH}$ is a professor in the Department of Frontier Surgery, Graduate School of Medical and Pharmaceutical Science, Chiba University, Chiba, Japan. SK is an associate professor in the Department of Surgery, Teikyo University Chiba Medical Center, Chiba, Japan. KC and MM are assistant professors in the Department of Surgery, Teikyo University Chiba Medical Center, Chiba, Japan. NK is a staff member in the Department of Surgery, Teikyo University Chiba Medical Center, Chiba, Japan.

\section{Ethics approval and consent to participate}

The study was conducted in accordance with the principals of the Declaration of Helsinki. The Teikyo University Chiba Medical Center and Chiba University Hospital Ethics Review Boards approved retrospective data collection and analysis. All patients gave written informed consent for the collection of their medical data for scientific purposes

\section{Consent for publication}

Not applicable.

\section{Competing interests}

The authors declare that they have no competing interests.

\section{Publisher's Note}

Springer Nature remains neutral with regard to jurisdictional claims in published maps and institutional affiliations.

\section{Author details}

'Department of Surgery, Teikyo University Chiba Medical Center, 3426-3 Anesaki, Ichihara, Chiba 299-0111, Japan. ${ }^{2}$ Center for Frontier Medical Engineering, Chiba University, Chiba, Japan. ${ }^{3}$ Department of Frontier Surgery, Graduate School of Medical and Pharmaceutical Science, Chiba University, Chiba, Japan.

\section{Received: 1 June 2018 Accepted: 2 December 2018}

Published online: 17 December 2018

\section{References}

1. Ferlay J, Soerjomataram I, Dikshit R, Eser S, Mathers C, Rebelo M, et al. Cancer incidence and mortality worldwide: sources, methods and major patterns in GLOBOCAN 2012. Int J Cancer. 2015;136:E359-86.
2. Sasako M, Sakuramoto $S$, Katai $H$, Kinoshita T, Furukawa $H$, Yamaguchi T, et al. Five-year outcomes of a randomized phase III trial comparing adjuvant chemotherapy with S-1 versus surgery alone in stage II or III gastric cancer. J Clin Oncol. 2011;33:4387-93.

3. Bang YJ, Kim YW, Yang HK, Chung HC, Park YK, Lee KH, et al. Adjuvant capecitabine and oxaliplatin for gastric cancer after D2 gastrectomy (CLASSIC): a phase 3 open-label, randomised controlled trial. Lancet. 2012; 379:315-21.

4. Shitara K, Chin K, Yoshikawa T, Katai H, Terashima M, Ito S, et al. Phase II study of adjuvant chemotherapy of S-1 plus oxaliplatin for patients with stage III gastric cancer after D2 gastrectomy. Gastric Cancer. 2017;20:175-81.

5. Cancer Genome Atlas Research Network. Comprehensive molecular characterization of gastric adenocarcinoma. Nature. 2014;513:202-9.

6. Coussens LM, Werb Z. Inflammation and cancer. Nature. 2002;420:860-7.

7. Leitch EF, Chakrabarti M, Crozier JE, McKee RF, Anderson JH, Horgan PG, et al. Comparison of the prognostic value of selected markers of the systemic inflammatory response in patients with colorectal cancer. Br J Cancer. 2007; 97:1266-70

8. Smith RA, Bosonnet L, Raraty M, Sutton R, Neoptolemos JP, Campbell F, et al. Preoperative platelet-lymphocyte ratio is an independent significant prognostic marker in resected pancreatic ductal adenocarcinoma. Am J Surg. 2009;197:466-72.

9. Teramukai S, Kitano T, Kishida Y, Kawahara M, Kubota K, Komuta K, et al. Pretreatment neutrophil count as an independent prognostic factor in advanced non-small-cell lung cancer: an analysis of Japan multinational trial organisation LC00-03. Eur J Cancer. 2009;45:1950-8.

10. Aliustaoglu M, Bilici A, Ustaalioglu BB, Konya V, Gucun M, Seker M, et al. The effect of peripheral blood values on prognosis of patients with locally advanced gastric cancer before treatment. Med Oncol. 2010;27:1060-5.

11. Shimada H, Takiguchi N, Kainuma O, Soda H, Ikeda A, Cho A, et al. High preoperative neutrophil-lymphocyte ratio predicts poor survival in patients with gastric cancer. Gastric Cancer. 2010;13:170-6.

12. Jung MR, Park YK, Jeong O, Seon JW, Ryu SY, Kim DY, et al. Elevated preoperative neutrophil to lymphocyte ratio predicts poor survival following resection in late stage gastric cancer. J Surg Oncol. 2011;104:504-11.

13. Lee S, Oh SY, Kim SH, Lee JH, Kim MC, Kim KH, et al. Prognostic significance of neutrophil lymphocyte ratio and platelet lymphocyte ratio in advanced gastric cancer patients treated with FOLFOX chemotherapy. BMC Cancer. 2013;13:350.

14. Jin H, Zhang G, Liu X, Liu X, Chen C, Yu H, et al. Blood neutrophillymphocyte ratio predicts survival for stages III-IV gastric cancer treated with neoadjuvant chemotherapy. World J Surg Oncol. 2013;11:112.

15. Min KW, Kwon MJ, Kim DH, Son BK, Kim EK, Oh YH, et al. Persistent elevation of postoperative neutrophil-to-lymphocyte ratio: a better predictor of survival in gastric cancer than elevated preoperative neutrophil-to-lymphocyte ratio. Sci Rep. 2017;7:13967. https://doi.org/10.1038/s41598-017-13969-x.

16. Balkwill F, Mantovani A. Inflammation and cancer: Back to Virchow? Lancet. 2001;357:539-45.

17. Mantovani A, Allavena P, Sica A, Balkwill F. Cancer-related inflammation. Nature. 2008;454:436-44

18. Dunn GP, Old LJ, Schreiber RD. The immunobiology of cancer immunosurveillance and immunoediting. Immunity. 2004;21:137-48.

19. Martínez-Lostao L, Anel A, Pardo J. How do cytotoxic lymphocytes kill cancer cells? Clin Cancer Res. 2015;21:5047-56.

20. Kao SC, Pavlakis N, Harvie R, Vardy JL, Boyer MJ, van Zandwijk N, et al. High blood neutrophil-to-lymphocyte ratio is an indicator of poor prognosis in malignant mesothelioma patients undergoing systemic therapy. Clin Cancer Res. 2010;16:5805-13.

21. Chua W, Charles KA, Baracos VE, Clarke SJ. Neutrophil/lymphocyte ratio predicts chemotherapy outcomes in patients with advanced colorectal cancer. Br J Cancer. 2011;104:1288-95.

22. Sato H, Tsubosa Y, Kawano T. Correlation between the Pretherapeutic neutrophil to lymphocyte ratio and the pathologic response to neoadjuvant chemotherapy in patients with advanced esophageal cancer. World J Surg. 2012;36:617-22.

23. Templeton AJ, Knox JJ, Lin X, Simantov R, Xie W, Lawrence N, et al. Change in neutrophil-to-lymphocyte ratio in response to targeted therapy for metastatic renal cell carcinoma as a prognosticator and biomarker of efficacy. Eur Urol. 2016;70:358-64.

24. Ohno Y, Nakajima J, Ohori M, Gondo T, Hatano T, Tachibana M. Followup of neutrophil-to-lymphocyte ratio and recurrence of clear cell renal cell carcinoma. J Urol. 2012;187:411-7. 
25. Jin F, Han A, Shi F, Kong L, Yu J. The postoperative neutrophil-to-lymphocyte ratio and changes in this ratio predict survival after the complete resection of stage I non-small cell lung cancer. Onco Targets Ther. 2016;9:6529-37.

26. Kang M, Jeong CW, Kwak C, Kim HH, Ku JH. The prognostic significance of the early postoperative neutrophil-to-lymphocyte ratio in patients with urothelial carcinoma of the bladder undergoing radical cystectomy. Ann Surg Oncol. 2016;23:335-42.

27. Lohrisch C, Paltiel C, Gelmon K, Speers C, Taylor S, Barnett J, et al. Impact on survival of time from definitive surgery to initiation of adjuvant chemotherapy for early-stage breast cancer. J Clin Oncol. 2006;24:4888-94.

28. Fairfield KM, Murray K, Lucas FL, Wierman HR, Earle CC, Trimble EL, et al. Completion of adjuvant chemotherapy and use of health services for older women with epithelial ovarian cancer. J Clin Oncol. 2011;29:3921-6.

29. Aspinall SL, Good CB, Zhao X, Cunningham FE, Heron BB, Geraci M, et al. Adjuvant chemotherapy for stage III colon cancer: relative dose intensity and survival among veterans. BMC Cancer. 2015;15:62.

30. Matsumoto I, Tanaka M, Shirakawa S, Shinzeki M, Toyama H, et al. Postoperative serum albumin level is a marker of incomplete adjuvant chemotherapy in patients with pancreatic ductal adenocarcinoma. Ann Surg Oncol 2015;22:2408-2415.

31. Aoyama T, Yoshikawa T, Shirai J, Hayashi T, Yamada T, Tsuchida K, et al. Body weight loss after surgery is an independent risk factor for continuation of S-1 adjuvant chemotherapy for gastric cancer. Ann Surg Oncol. 2013;20:2000-6.

32. Kimura M, Go M, Iwai M, Usami E, Teramachi H, Yoshimura T. Usefulness of a pharmacist outpatient service for S-1 adjuvant chemotherapy in patients with gastric cancer. Mol Clin Oncol. 2017;7:486-92.

Ready to submit your research? Choose BMC and benefit from:

- fast, convenient online submission

- thorough peer review by experienced researchers in your field

- rapid publication on acceptance

- support for research data, including large and complex data types

- gold Open Access which fosters wider collaboration and increased citations

- maximum visibility for your research: over $100 \mathrm{M}$ website views per year

At $\mathrm{BMC}$, research is always in progress.

Learn more biomedcentral.com/submissions 